\title{
Información relevante que pasa a ser anecdótica: aerinita en las pinturas murales románicas de San Martín de Elines (Cantabria)
}

\author{
Ma Rosa Tera Saavedra y Enrique Parra Crego
}

Resumen: Ante la dificultad de consultar datos materiales y analíticas provenientes de intervenciones existentes relacionadas con el Patrimonio Cultural, el presente artículo plantea la necesidad de establecer parámetros normalizados y accesibles que aglutinen información de memorias técnicas.

Como ejemplo de la funcionalidad que podría derivarse de esta propuesta se analiza cómo podría beneficiar su uso en el caso de las pinturas románicas de San Martín de Elines (Cantabria) cuya analítica de materiales presenta dos parámetros fuera del contexto geográfico donde están ubicadas y que las relaciona matéricamente con las pinturas románicas del Valle del Boí.

Palabras clave: Contexto; normalización; pintura mural románica; aerinita

\section{Blue pigment of the Romanesque paintings of San Martin de Elines (Cantabrian Region, North of Spain)}

Abstract: Given the difficulty of consult material data and analytics from existing interventions related to the Cultural Heritage, this article raises the need for standardized and accessible parameters which bring together information from technical reports.

As an example of the functionality that could result from this proposal is analyzed how its use could benefit in the event of Romanesque paintings of San Martín de Elines (Cantabria) whose analytical of materials presents two parameters outside the geographical context where they are located and whose matter is related with the Boí Valley Romanesque paintings.

Key words: Context; normalitation; Romanesque mural paintings; aerinita

\section{Informação relevante que passou a ser anedótica : Aerinite nas pinturas murais românicas de San Martin de Elines ( Cantabria)}

Resumo: Perante a dificuldade de consultar dados materiais e análises provenientes de intervenções existentes relacionadas com o Património Cultural, o presente artigo coloca a necessidade de estabelecer parâmetros normalizados e acessíveis que aglutinem informação de relatórios técnicos.

Como exemplo da funcionalidade que poderia resultar como consequência desta proposta, analisa-se como se poderia beneficiar da sua utilização no caso das pinturas românicas de San Martin de Elines (Cantabria) cuja análise dos materiais apresenta dois parâmetros fora do contexto geográfico das obras mencionadas e que as relaciona materialmente com as pinturas românicas do Vale do Boí.

Palavras-chave: Contexto, normalização, pintural mural romanica, aerinite 


\section{Introducción}

El motivo de este trabajo surge ante la carencia y necesidad de implementación, técnica y administrativa, para normalizar y relacionar gran cantidad de información existente, fruto de innumerables intervenciones de conservación-restauración.

Su propósito es el de proponer la necesidad de una metodología, estructurada en forma de base de datos, que sirva para relacionar parámetros y que permita contextualizar Patrimonio Cultural y a modo de ejemplo pintura mural románica, a partir del cotejo de sus materiales constructivos y técnica de ejecución.

La necesidad de esta herramienta se justifica ante la ampliación conceptual de los objetivos de las intervenciones que han pasado de ser meras acciones de conservación-restauración a ser actividades que desarrollan información inestimable sobre procesos y materiales de ejecución que han quedado registradas en memorias técnicas. Esta falta de normalización atañe, en general, a los Bienes Culturales a pesar de que en su intervención imperan criterios de restauración que han de ceñirse a leyes, cartas y recomendaciones relacionadas con la defensa del Patrimonio.

\section{Antecedentes del trabajo de investigación}

En julio de 2010 el párroco de la Colegiata de San Martín de Elines, ubicada en el Valle de Valderredible (Cantabria), don Bertín Gutiérrez López, promueve la intervención de las pinturas murales románicas que se conservan en la cabecera del templo.

La propuesta queda aprobada según informe emitido por la Dirección General de Bellas Artes y Bienes Culturales del Ministerio de Cultura, como indica el acuerdo remitido por la Consejería de Cultura, Turismo y Deporte del Gobierno de Cantabria con fecha 22 de Junio de 2011 y no de registro 4544. En dicho informe se "insta a que la empresa revise los extremos relativos a la técnica de ejecución, las dimensiones y materiales y sugiere la realización de una memoria histórico-artística de mayor profundidad por el carácter único de dichas pinturas murales en Cantabria", es decir, se solicita que la intervención no se limite al tratamiento directo en el bien, sino que establezcan las medidas oportunas para situar la obra en un determinado contexto.

Ante la falta de fuentes documentales de referencia y con el objeto de cumplir la instancia se desarrolla un trabajo de investigación en el que se muestra brevemente el contexto político-social de la época de ejecución de obra, se aborda su ficha técnica y se realiza una descripción histórico-artística e iconográfica de la misma. Este desarrollo muestra cómo pueden y suelen enfocarse este tipo de análisis en los informes de intervención con los medios que actualmente se encuentran a disposición de los conservadores-restauradores. Sin embargo, debido a la falta de normalización y accesibilidad a la información no es posible realizar un estudio comparativo de la información que en ellos se recoge.

El presente artículo, a través del ejemplo de los datos obtenidos en la intervención de las pinturas murales románicas de San Martín de Elines, es prueba de ello. Muestra que el análisis de materiales, en una obra carente de fuentes documentales de referencia, puede definir peculiaridades y establecer relaciones con otras similares. Sin embargo, llegados a este punto es donde comienzan los problemas. Estos planteamientos metodológicos que implican la realización de un estudio matérico e históricoartístico exhaustivo no siempre es factible, debido a inconvenientes económicos, a lo que hay que añadir la falta de conocimiento sobre otros estudios que hayan sido realizados en los Servicios de Patrimonio de las respectivas consejerías, que por otra parte no aparecen en un formato normalizado de consulta.

En la siguiente imagen [Tabla 1] se muestra la ficha técnica de estas pinturas murales en la que de manera esquemática se recoge la información más relevante de la misma.

\section{Datos de una intervención que ayudan a establecer contexto}

Los aspectos de mayor relevancia que pueden ayudar a establecer relaciones, si se habla de contextos carentes de fuentes documentales, son el análisis material, histórico y artístico de la obra.

En una visión transversal de lo que puede suponer una intervención de conservación-restauración, observamos que sus objetivos suelen superar con creces la simple intervención material. El establecimiento del objeto en un contexto cultural se hace imprescindible para su comprensión, disfrute y por lo tanto su preservación. Para establecer esta relación, el punto de partida de la intervención material correctamente ejecutada debe, al menos, aspirar a ser capaz de plantear una serie de correspondencias que posteriormente facilitarán y quizás permitirán el estudio y el adecuado cotejo de unos bienes con otros.

Como precedente a la intervención la documentación histórico-artística de la obra constituye una ayuda para el profesional encargado de esta labor. Y a su vez, la información obtenida por el restaurador durante su labor, sin duda, podrá complementar la desarrollada por el historiador y el laboratorio encargado de los análisis de la materia constituyente de la obra.

A continuación se mostrarán los datos obtenidos en el caso tomado como ejemplo. 


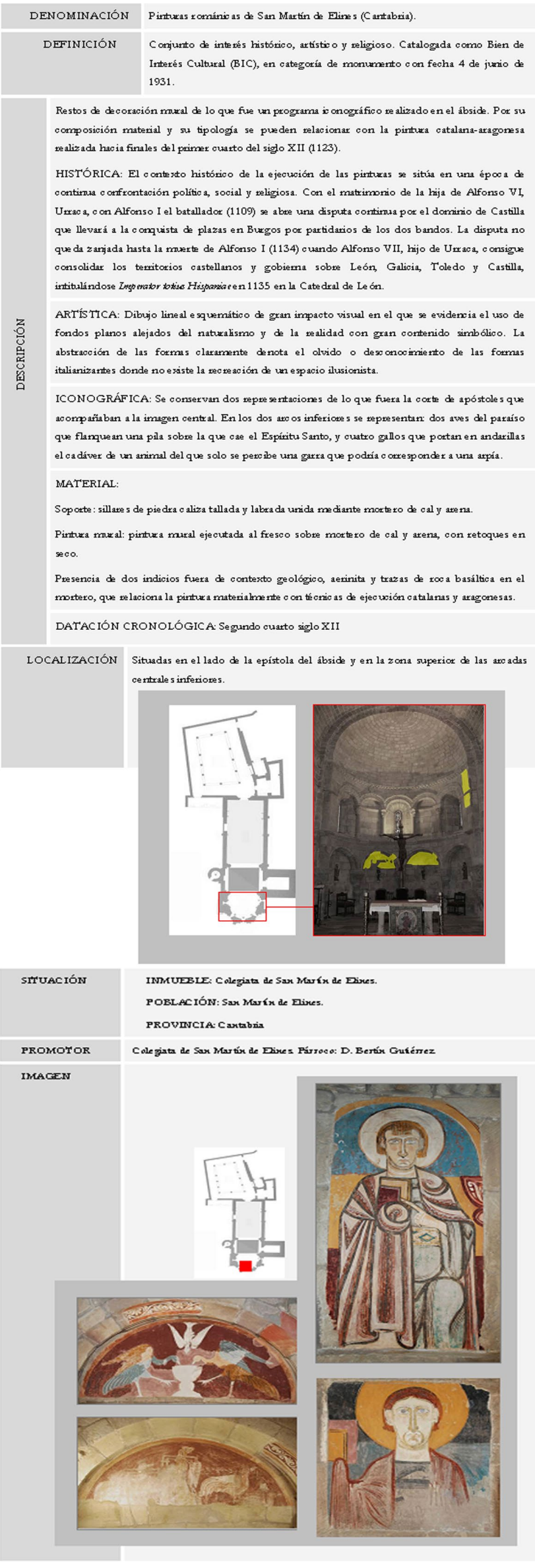

Tabla 1.- Ficha técnica de las pinturas murales de San Martín de Elines. @Rosa Tera.

\section{—Datos históricos}

La ejecución de las pinturas que sirven de ejemplo se produce en un contexto histórico de continuo cambio. Muchas son las preguntas que se podrían formular sobre la iglesia de San Martín de Elines: ¿A qué centro político, económico, cultural o artístico respondía?, ¿quién fue el promotor del templo?, ¿y de las pinturas?, ¿por qué las características estilísticas de los restos de sus pinturas se puede relacionar con determinados conjuntos de la pintura catalana-aragonesa? Aunque pocos son los datos que existen para establecer estas respuestas se puede comentar lo siguiente para encuadrarlas en un conjunto.

A lo largo de los siglos XI y siglo XII fue notable la consagración de nuevos templos en ámbitos rurales como lo indican las actas de consagración y dotaciones existentes en todo el ámbito cristiano.

Ante la escasez de fuentes documentales relacionadas con las citadas pinturas murales, y con el objeto de establecer un encuadre histórico, se mencionarán determinados aspectos que se consideran relevantes para intentar definir las relaciones que podían existir en el siglo XII entre zonas alejadas como Castilla y los condados catalanes. Se incidirá cronológicamente en la época posterior a 1123, fecha de consagración de las iglesias románicas de Santa María y de Sant Climent de Taüll en el valle de Boí (Alta Ribagorça, Lleida), por considerar dichas pinturas como referentes a nivel tipológico y material, de que nos ocupan en esta investigación, situadas en el valle de Valderredible (Cantabria).

Como precedente, mencionar que durante el siglo XI la política de reconquista de territorios taifales por parte de los cristianos pone de manifiesto que sólo es posible establecer posiciones de defensa, y que se carece de medios suficientes para la derrota definitiva debido a la escasez, principalmente, de recursos humanos.

En el territorio de Aragón este tipo de planteamientos comienza a variar con la figura de Alfonso I el batallador (1104-1134), criado en el monasterio de Siresa (valle de Hecho, Huesca) quien, basándose en privilegios otorgados o fueros, establece cambios para estimular una nueva legislación. Concede privilegios ventajosos a quien le ayude militarmente, formando cuerpos de caballería no nobiliaria, funda órdenes propias y consigue apoyo de nobles del otro lado de los Pirineos mediante "Bulas de Cruzada".

En Castilla, a la muerte de Sancho hijo y sucesor del rey Alfonso $\mathrm{VI}$ de León, el monarca convoca a los nobles para que nombren sucesora a su hija Urraca. Esta, viuda de Raimundo de Borgoña y madre de Alfonso Raimúndez, es aceptada con la condición de que contraiga nuevo matrimonio inmediatamente. Ante las rivalidades de castellanos y leoneses a favor de unos u otros pretendientes, el rey decide casarla con Alfonso I de Aragón, celebrándose el matrimonio en 1109 en el Castillo de Monzón de Campos (Palencia). Esta unión provocará un conflicto continuado 
con la oposición del clero, obispos y nobles'1, formándose dos tendencias. Por una parte los partidarios de Alfonso I, que integran a la baja nobleza y a las grandes ciudades que bordean el Camino de Santiago que están deseosas de deshacerse de los señoríos eclesiásticos. Por otra los partidarios de Urraca formados por la alta nobleza y el clero que no desean grandes cambios estructurales en la forma de gobernanza. Esta relación, de constante confrontación política, genera continuos asedios en plazas castellanas que son tomadas por uno u otro bando. En este contexto político conviene mencionar que la plaza de Burgos y otras ciudades cercanas2 son tendentes a Alfonso I.

Centrándonos en el caso que nos ocupa, Valderredible figura pronto como una unidad territorial diferenciada aunque más tarde se junte con Campoo y ambas pasen a formar la merindad de Aguilar de Campoo, si bien conservan singularidades por su carácter de valle. Desde el siglo IX se encuentran dentro del condado de Castilla y dependen de la diócesis de Burgos. En el siglo XII las organizaciones administrativas y políticas se complican al reforzarse consejos y hermandades.

Según García Guinea (García y Pérez 2007:1426), Luciano Serrano (1936) comenta que la colegiata era de patronato particular y estaba sometida a jurisdicción de la diócesis de Burgos. Los patronos eran los encargados de nombrar al abad, tras ser presentado previamente al diocesano, y la elección de los canónigos la realizaban entre el patrono y el abad. El Cabildo gozaba de autonomía interna, y el abad nombraba a los clérigos de las iglesias dependientes de la colegiata. Cuenta que en 1165, Alfonso VIII daba a Pedro Martínez, abati de Nelines, las villas de Espinosilla y Repentidos, en el alfoz de Rabanales, según carta de donación y confirmación.

\section{-Descripción artística}

En relación a la cronología y datación, los conocimientos sobre pintura mural románica no son suficientemente amplios como para estructurar una teoría congruente sobre la misma. Se puede afirmar que el periodo central de la producción pictórica románica es el siglo XII.

La pintura que nos ocupa muestra un dibujo lineal alejado del naturalismo y de la realidad. No existe recreación de un espacio ilusionista. La representación adquiere únicamente valores bidimensionales y se estructuran los fondos mediante franjas monocromas de color, superpuestas horizontalmente sin solución de continuidad, de manera que sigue el modelo utilizado en los Beatos.

En su ejecución es común la superposición de capas pictóricas, algunas incluso con cierto empaste en las pinceladas. El tratamiento anatómico de rostros se realiza con líneas en negro y blanco y se moldean ciertos detalles, como las mejillas, con veladuras más rojas. Las líneas negras actúan como delimitador y estructurador de las formas y las blancas dan viveza y calidad de brillo a las figuras.

En la búsqueda de referencias estilísticas, para el ejemplo de Elines, no dejan de sorprender las similitudes tipológicas relacionadas con restos de pinturas románicas realmente alejadas geográficamente [figura 1]. Se puede observar claramente la coincidencia en la forma de la cabeza de uno de los apóstoles de San Martín de Elines con uno de la ermita de la Veracruz de Maderuelo (Segovia) y con los restos de los apóstoles procedentes de la colegiata de la canónica de Sant Pere d'Àger (Noguera, Lleida).

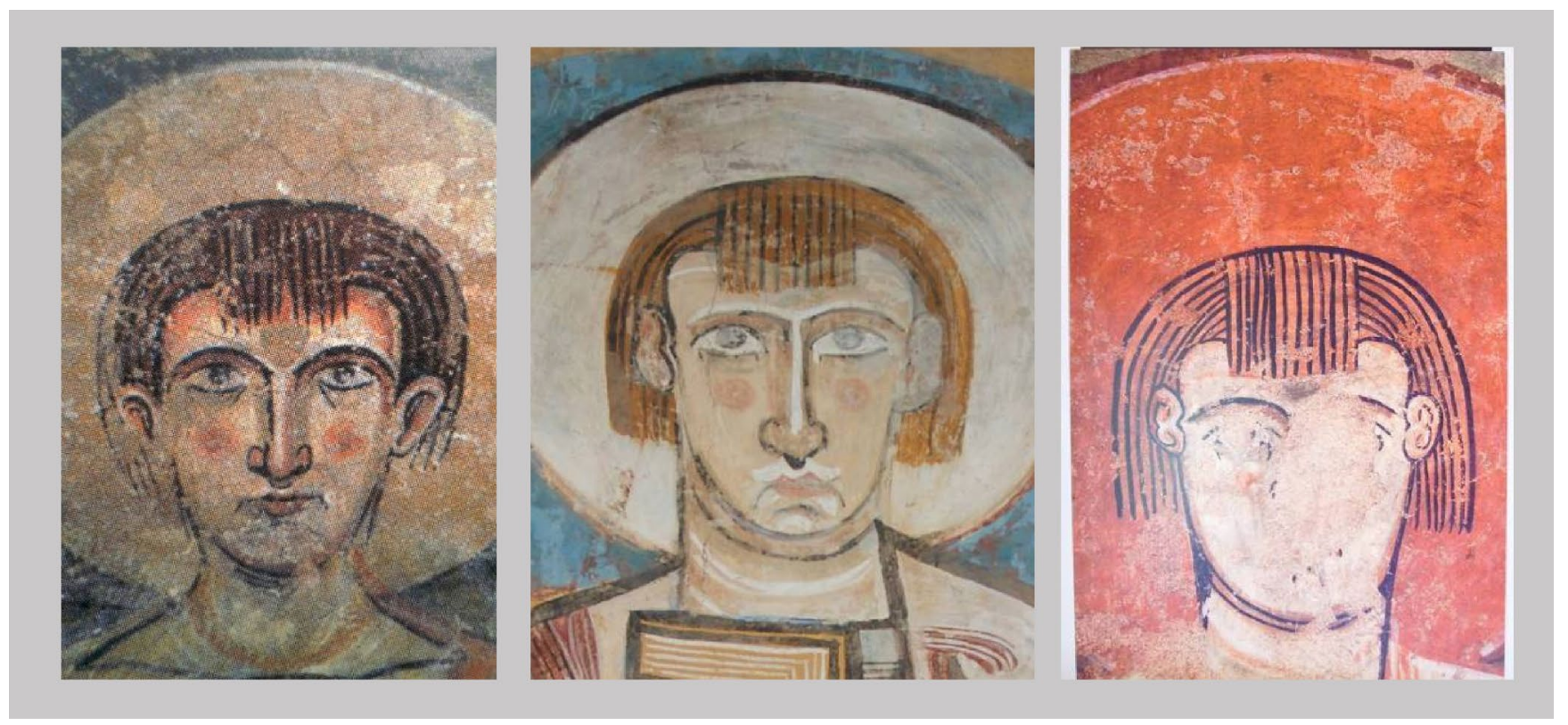

Figura 1.- Comparaciones estilísticas en cuanto a estructura de frontalidad y solución. de izquierda a derecha: representaciones de Àger (MNAC), procedentes del Monasterio de Sant Pere (Noguera, Lleida), de la colegiata de San Martín de Elines (Cantabria) y de la ermita de la Veracruz en Maderuelo (Segovia). ORosa Tera. 
Otras coincidencias existentes con Santa María de Taüll (Lleida), son las franjas azules que aparecen sobre fondos rojos, que han sido utilizados para definir la composición, y el empleo de líneas de contorno negras y blancas. En este aspecto sería sumamente interesante definir si ambas están realizadas con la misma composición material.

Otras representaciones de pintura mural románica en zonas próximas a San Martín de Elines (Cantabria) existían hasta 1957 en un muro del campanario de la iglesia románica de San Miguel en Tubilla del Agua (Burgos). Sureda las relaciona con San Martín de Elines. Por lo que respecta a su cronología, Gaya Nuño las consideró posteriores, de la segunda mitad del siglo XII, Post advirtió ciertas relaciones con los murales de Pedret y Ainaud la relacionó con el taller de Taüll (Huerta 2004: 206).

Aunque hay evidencias tipológicas similares entre diversos ciclos como Roda de Isábena (Huesca), la ermita de San Baudelio de Casillas de Berlanga (Soria), la ermita de la Santa Cruz de Maderuelo (Segovia), el fragmento de Tubilla del Agua (Burgos) y los restos de San Martín de Elines (Cantabria), no se pretende decir con esto que sean obras del mismo artista, sino que representan la continuidad de un modo estilístico que parece dominante en los reinos hispánicos hasta la mitad del siglo XII y de los que, fuera de Cataluña, han quedado mayor abundancia de restos conservados. La expansión de esta manera estilística en lugares tan distantes puede indicar que existió una razón superior a la movilidad de artistas, que motivase esta expansión [figura 2].

Relacionando las evidencias materiales con los hechos históricos, se llega a la conclusión de que no existen datos suficientes para establecer hasta qué grado la figura de Alfonso I el batallador puede ser una figura clave en el proceso. Sin embargo, todas las iglesias anteriormente mencionadas, cuyas representaciones se asemejan a

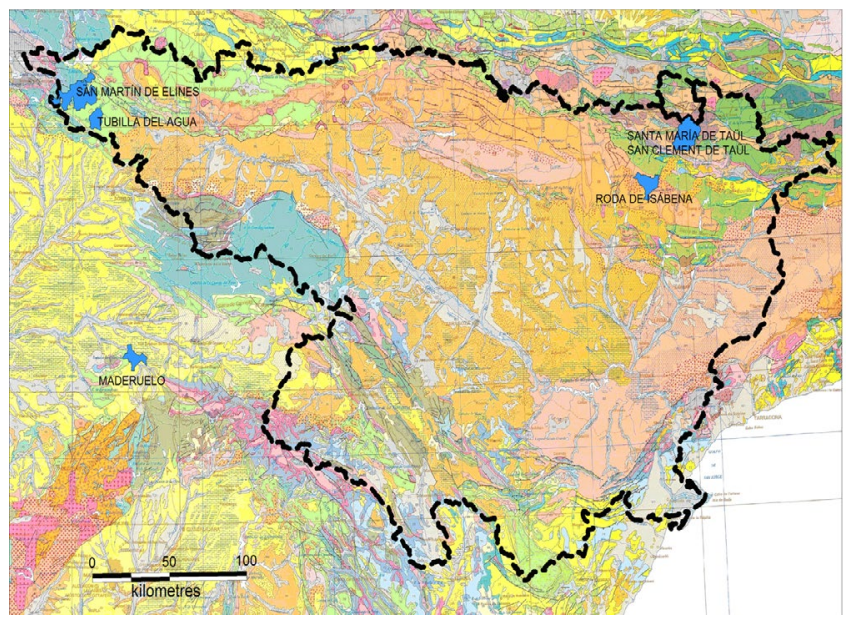

Figura 2.- Localización territorial de la cuenca del Ebro. Se puede observar que muchas de las pinturas rómánicas mencionadas se inscriben en ella. Fuente:Bárbara Quevedo Pangéa Proyectos . tipologías catalano-aragonesas, pertenecen a la zona de influencia aragonesa incluso después de la muerte de Urraca.

\section{-Descripción material}

La pintura que nos ocupa está realizada al fresco ejecutado sobre argamasa de cal y arena, según el procedimiento descrito por Theophilus en su tratado Diversarum Artium Schedule. Las técnicas analíticas utilizadas en la caracterización de los materiales han sido microscopía óptica (MO), microscopía electrónica de barrido (MEB), espectroscopía infrarroja por transformada de Fourier (FTIR) y cromatografía de gases (CG).

Para el análisis por $\mathrm{MO}$ se ha utilizado un microscopio petrográfico Olympus BX60, trabajando entre 50 y 500 $X$ y equipado con sistema de microfotografía. Para el estudio de las muestras, éstas se incluyeron en resina acrílica PRESI-KMU y se prepararon cortes transversales. Las microfotigrafías se obtuvieron con luz reflejada, a 300X y con nícoles cruzados. Las tinciones selectivas de proteínas se realizaron con fuchsina ácida y con negro amido II, dependiendo de los colores de los pigmentos presentes en cada muestra. Las tinciones selectivas para la detección preliminar de aceites, se llevaron a cabo con negro Sudán $\mathrm{S}$.

Las preparaciones y la superficie de la pintura, así como el interior de algunas de las capas pictóricas fueron analizadas mediante espectroscopía FTIR usando un espectrómetro Spectrum One de Perkin Elmer con accesorio de reflectancia total atenuada universal (U-ATR). Los espectros se realizaron entre 550 y $4000 \mathrm{~cm}^{-1}$ trabajando siempre a 4 cm-1 de resolución.

El análisis de pigmentos y granos de mineral se llevó a cabo combinando la información del microscopio óptico con el análisis mediante microscopía electrónica de barrido acoplada a microanálisis de energía dispersiva de rayos $X$ (MEB/EDX) que se llevó a cabo sobre las propias estratigrafías, previo su recubrimiento con grafito. El análisis se realizó en un microscopio Hitachi S-3000N con detector EDX trabajando hasta $50000 \mathrm{X}$.

Para el análisis de aglutinantes las muestras fueron analizadas con un equipo de cromatografía de gases Perkin Elmer Clarus 500). Para la determinación de sustancias lipófilas, las muestras fueron tratadas con el reactivo de metilación Meth-prep Il; para la determinación de proteínas fueron sometidas a hidrólisis con $\mathrm{HCl} 6 \mathrm{M}$ y derivatización con tert-butildimetilsililtrifluoroacetamida (TBDMSTFA) en piridina de los ácidos grasos y aminoácidos.

Según los resultados analíticos obtenidos [figura 3 y tablas 2 y 3] la pintura fue ejecutada sobre un mortero de cal y arena que contiene calcita en la matriz aglomerante, con trazas de dolomita y en el árido, granos de cuarzo y roca basáltica molida, o arena basáltica. La proporción calcita 

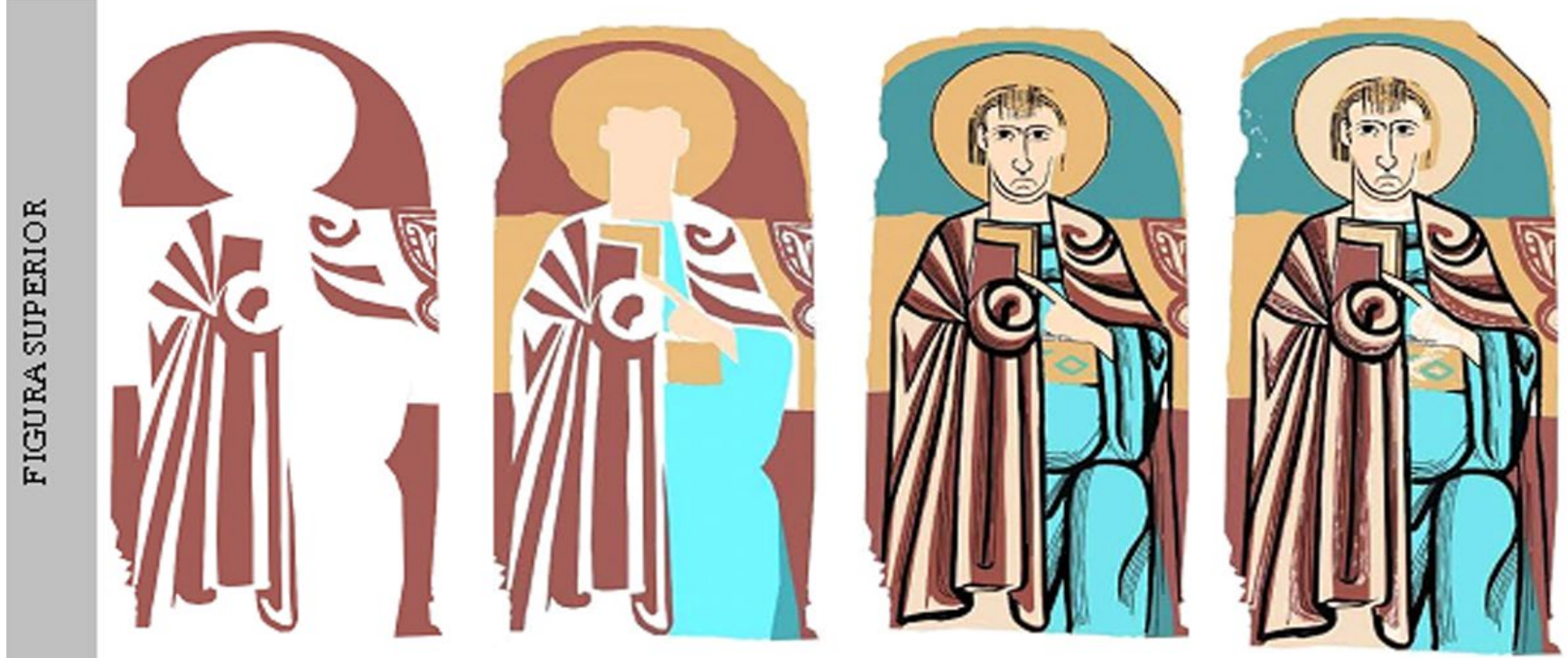

1'Fresco

2) Freso

$\left.3^{2}\right) \operatorname{Sec} 0$

4') Seco

Figura 3.- Secuencia y técnica de ejecución. @Rosa Tera

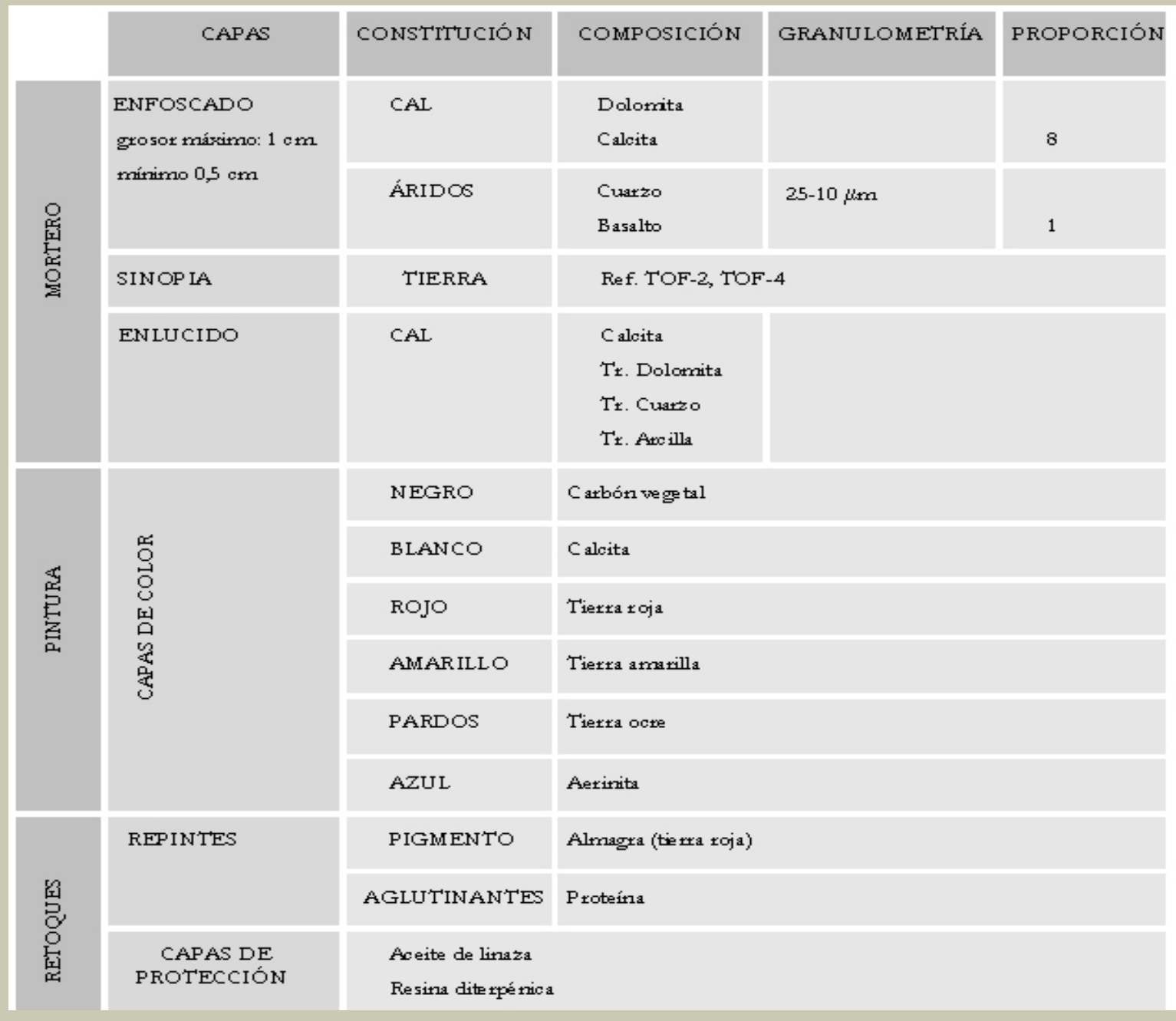

Tabla 2.- Resumen de la composición. @Rosa Tera 


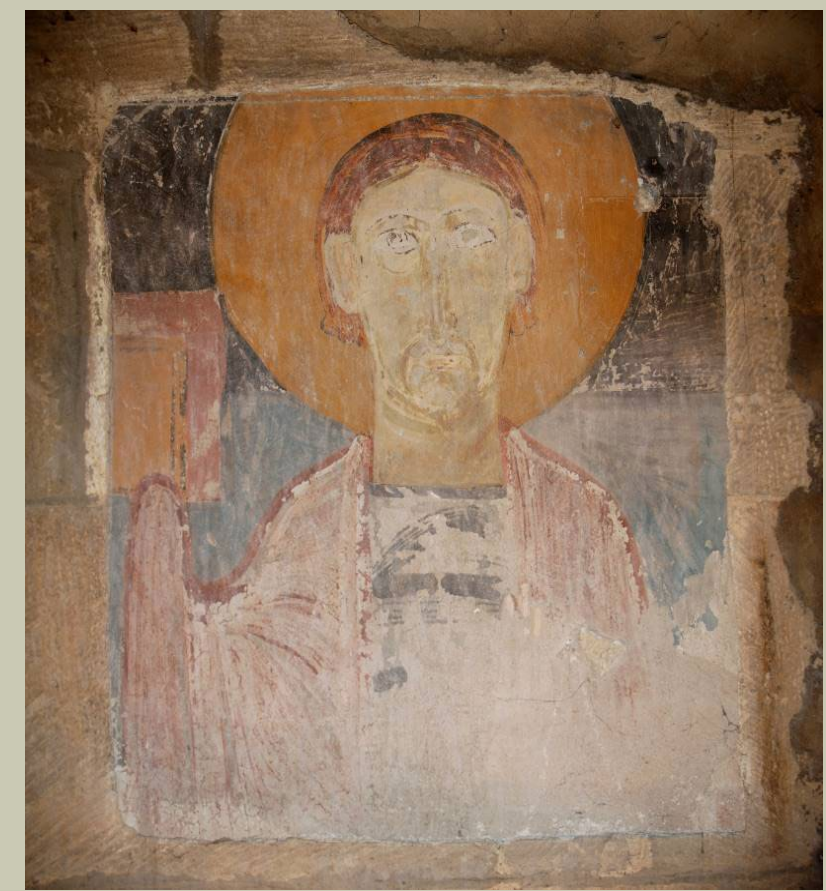

Muestra TOF-1 (Azul-Verdoso). @Rosa Tera

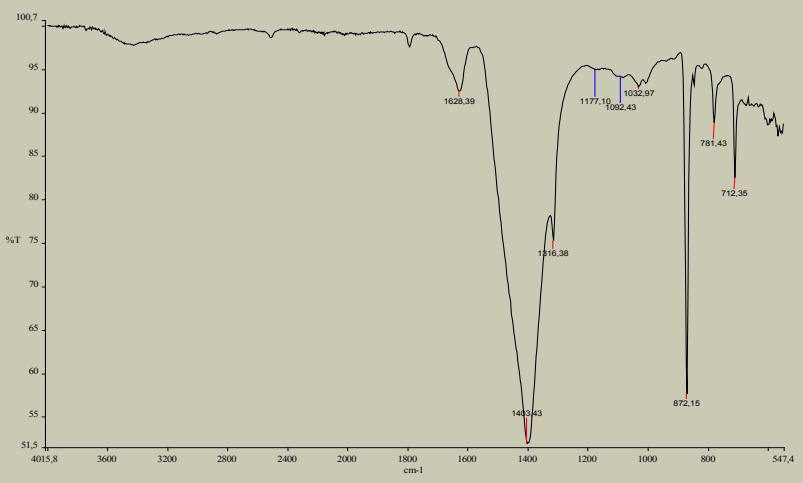

Preparación de la muestra TOF-1. Fuente: Enrique Parra

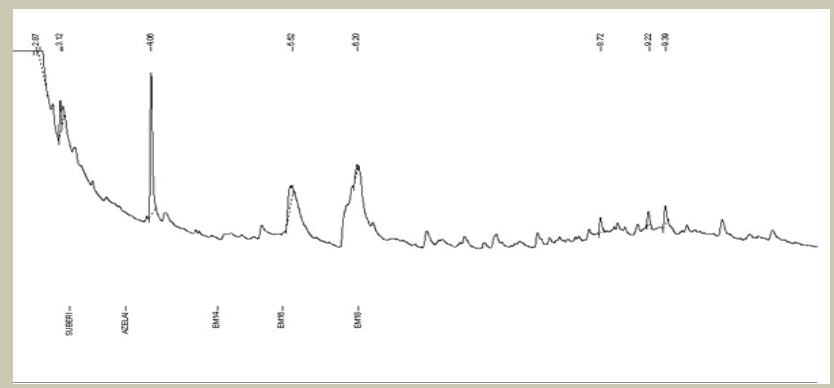

Cromatograma de metilación de ácidos grasos y diterpenos de la muestra TOF-1. Se detectan palmitato de metilo (EM16) y estearato de metilo (EM18), no aparecen el acelato (AZELAI) ni el suberato de metilo (SUBERI). Los diterpenos aparecen en torno a $9 \mathrm{~min}$. Todos estos componentes están a niveles muy bajos, por lo que pueden ser debidos a la contaminación que producen las protecciones aplicadas en tiempos pasados. (c) Enrique Parra.



Estratigrafía de la muestra TOF-1. La preparación contiene una matriz de calcita con un grano de cuarzo y otro de basalto (300 $\mathrm{X})$. @Enrique Parra

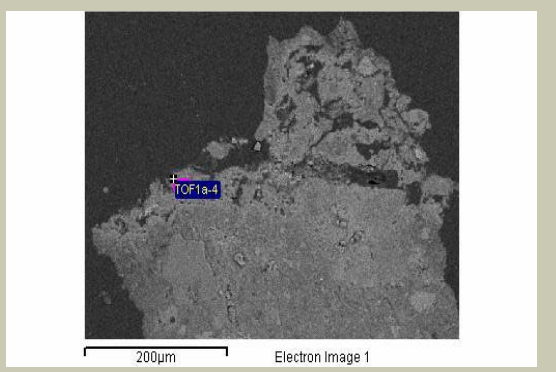

Microanálisis de un grano de pigmento azul de la capa pictórica de la muestra TOF-1. Imagen MEB, en medio espectro elemental. @Enrique Parra.

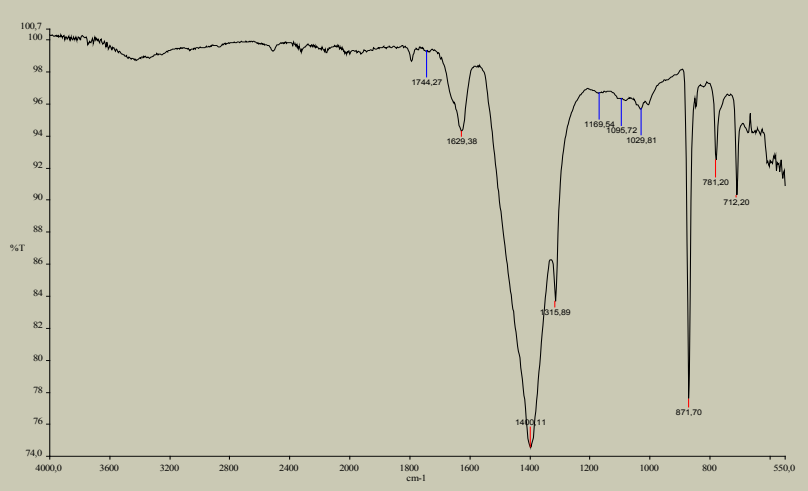

Superficie de la muestra TOF-1. Fuente: Enrique Parra

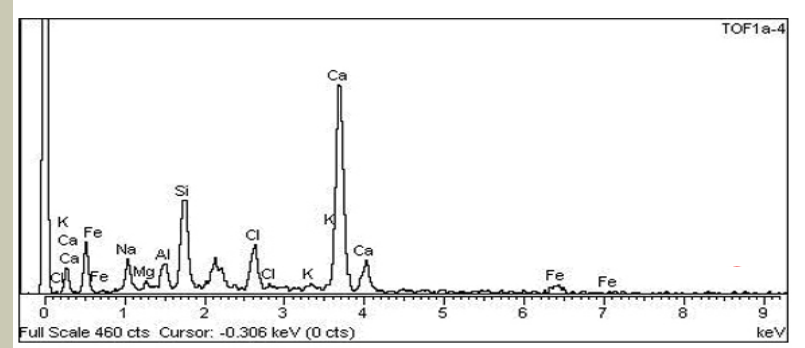

Espectro elemental de un grano de pigmento azul de la capa pictórica de la muestra TOF-1 Fuente: Enrique Parra 
Owner: INCA

Site: Site of Interest 2

Sample: TOF-1a

Type: Default

Processing option : All elements analyzed (Normalised)

Number of iterations $=3$
Standard :

Na Albite 1-Jun-1999 12:00 AM

Mg MgO 1-Jun-1999 12:00 AM

Al Al2O3 1-Jun-1999 12:00 AM

Si SiO2 1-Jun-1999 12:00 AM

CI KCI 1-Jun-1999 12:00 AM

K MAD-10 Feldspar 1-Jun-1999

12:00 AM

Ca Wollastonite 1-Jun-1999 12:00

AM

Fe Fe 1-Jun-1999 12:00 AM

\begin{tabular}{|c|c|c|c|c|c|}
\hline Element & App & Intensity & Weight $\%$ & Weight $\%$ & Atomic $\%$ \\
\hline & Conc. & Corrn. & & Sigma & \\
\hline $\mathrm{NaK}$ & 0.29 & 0.6583 & 1.07 & 0.70 & 1.67 \\
\hline $\mathrm{MgK}$ & 0.72 & 0.6506 & 2.68 & 0.67 & 3.97 \\
\hline $\mathrm{A} 1 \mathrm{~K}$ & 1.87 & 0.7578 & 6.00 & 0.72 & 8.00 \\
\hline SiK & 6.11 & 0.8121 & 18.28 & 1.03 & 23.39 \\
\hline $\mathrm{ClK}$ & 0.32 & 0.7458 & 1.04 & 0.55 & 1.05 \\
\hline $\mathrm{KK}$ & 0.42 & 1.1061 & 0.92 & 0.51 & 0.84 \\
\hline $\mathrm{Ca} \mathrm{K}$ & 25.75 & 0.9876 & 63.34 & 1.53 & 56.79 \\
\hline $\mathrm{Fe} \mathrm{K}$ & 2.24 & 0.8189 & 6.66 & 1.11 & 4.29 \\
\hline Totals & & & 100.00 & & \\
\hline
\end{tabular}

Caracterización del pigmento azul. Cuantificación de elementos mediante análisis elemental por energía dispersiva de rayos $X$ (ESEM/EDX). Fuente: Enrique Parra

Tabla 3.- Detalles analíticos de la muestra de pigmento . @Rosa Tera

/ cuarzo-silicatos es de 8/1. El tamaño de los granos de cuarzo es bastante regular y oscila entre 25 y $10 \mu \mathrm{m}$. Sobre la preparación, la pintura aparece sin aglutinar con materia orgánica por lo que se trata de un fresco. El pigmento azul ha sido identificado como aerinita (silicato de aluminio, magnesio y calcio) y se encuentra fuera del contexto geográfico de las pinturas, al igual que los fragmentos de roca basáltica de la capa de preparación.

Si analizamos la sucesión de capas de color de las diferentes escenas se puede determinar la secuencia y su técnica de ejecución [figura 3].

La primera capa corresponde a tonos rojizos utilizados como base y encaje del dibujo. No existen restos de incisiones, ni punteado que indique la forma utilizada para el encaje. En fresco, en contacto directo sobre el muro, se aplican los rojos de los fondos, las carnaciones y los verdes de la túnica. Sobre las capas de base descritas se aplica el color azul y los detalles de la dalmática, presumiblemente en seco -aunque no se ha realizado estratigrafía-, y se realizan las líneas negras sobre las que posteriormente se aplican pinceladas de blanco aglutinado con cola -según muestra estatigráfica- .

En el caso de la figura superior, su ejecución presenta las siguientes características:

- Capas rojizas preparatorias aplicadas a modo de base de otros colores.

- Las bases de las carnaciones se aplican en fresco quedando sin aplicar la zona de los ojos.

- Sobre los colores ejecutados al fresco se aplican colores en seco delimitando líneas y formas.

- La franja azul del fondo (aerinita) aparece aplicada sobre la capa rojiza anteriormente mencionada.

- La túnica verde probablemente se ha pintado bien mediante la mezcla de aerinita con pigmentos ocres, bien con aerinita verdosa -aunque no se ha realizado analítica-, o con tierra verde. 
- El color negro delinea la casi totalidad de la imagen, encontrándose en numerosos puntos tonos de brillos blancos sobre la misma línea negra, lo que nos indica la secuencia de aplicación.

Sin embargo, esta secuencia no es totalmente coincidente con la existente en la figura inferior, donde el fondo azul aparece aplicado directamente sobre el muro (no sobre la capa rojiza); las zonas ejecutadas en seco se limitan a los tonos blancos en las carnaciones, negros en los ojos y borde del nimbo; los ropajes carecen de delimitaciones en negro; y el nimbo es ocre, como el color existente bajo del nimbo de la figura superior [figura 4].

Las considerables diferencias en la ejecución nos indica que existen dos autores que trabajan en la obra con considerables variantes técnicas, cosa que no deja de ser peculiar puesto que en la mayoría de las obras las diferencias entre una mano y otra, no se hacen tan evidentes, pues suele existir un maestro que realiza una técnica y aprendices que la repiten.

Por otra parte, en el caso concreto de San Martín de Elines, los resultados de los análisis presentan dos extrañezas aparentemente fuera de contexto, que están en relación con la pintura románica alejada de este territorio y ejecutada en la zona catalano-aragonesa. Nos referimos a la presencia de aerinita y a la aparición de áridos de río de origen basáltico en el mortero de las pinturas.
El mineral llamado aerinita ha sido utilizado como pigmento azul en la pintura. Tradicionalmente fue usado como pigmento azul y verde entre los siglos XI y XV en gran número de pinturas románicas del ámbito pirenaico catalán, andorrano y francés fundamentalmente.

Químicamente la aerinita es un silicato azul asociado con la alteración de diabasas toleíticas (ofitas). En un estudio no publicado llevado a cabo en el Laboratorio de Mineralogía de la Universidad de Toulouse cuya referencia es UMR 5563 (Daniel et all 2008:83-91) se demuestra que la aerinita puede adquirir tonos verdes, aunque hay que destacar que la bibliografía en aerinita limita su estudio como pigmento azul (Palet y Andres 1992:132-136).

Las condiciones para su formación son muy especiales, por lo que los yacimientos se localizan únicamente en zonas próximas a los Pirineos españoles en: la provincia de Huesca, Aragón (Estropiñan del Castillo, Caserras, Juseu, Nasha); en Cataluña, en la provincia de Lleida (Tartareu, Villanueva de Avellanes, Ager, Artesa de Segre, Hostalets de Tosts); en Navarra; y Andalucía en la provincia de Málaga (Antequera). También se encuentran fuera de los Pirineos, donde otros depósitos se han descubierto en Marruecos, Atlas Medio y Anti-Atlas, en las afueras de la ciudad de Ouarzazate, en el norte de la Italia, Portugal (Serra Argo) y Estados Unidos (Arizona).

En la pintura del norte de España (Cataluña, Aragón, Andorra), este mineral ha sido identificado y reconocido por primera vez
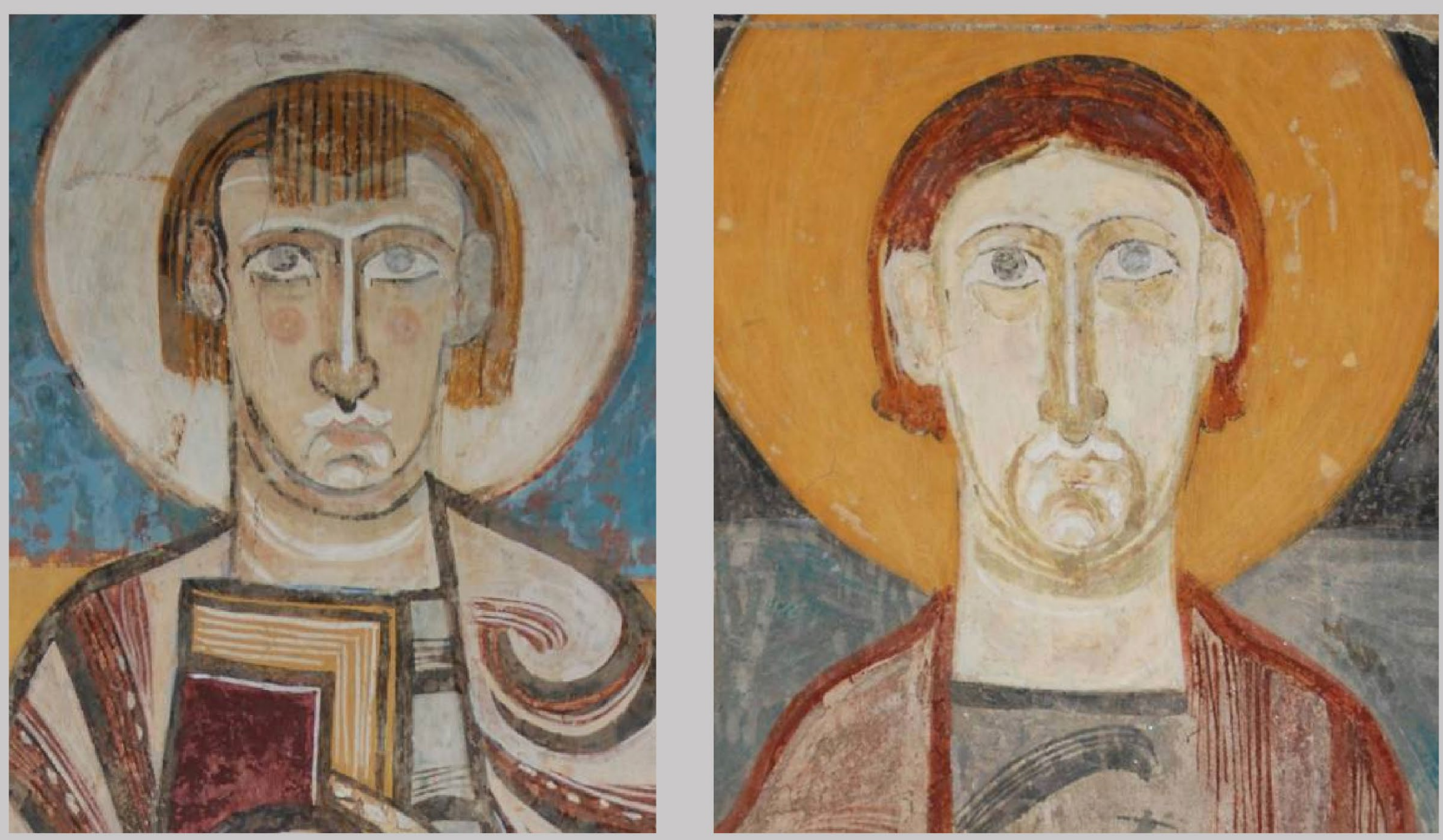

Figura 4.- Comparativa de la técnica de ejecución en las dos figuras existentes. 
en 1990 en decenas de pinturas murales románicas, entre las que se encuentran, citando sólo las más conocidas, las de Santa María de Taüll (Cataluña, siglo XII) o Santa María de Sigena (Aragón, siglo XII) que se conservan en el Museo Nacional de Arte de Cataluña (Porta, Palet y Guillamet 1990:534-538).

La utilización de este pigmento, sin embargo, va más allá del marco geográfico y cronológico indicado. En las intervenciones realizadas durante la conservación y restauración de las pinturas murales de la antigua abadía de Moissac (Tarn et Garonne, Francia, siglo XII) y la iglesia de Saint-Nicolas de Nogaro (Gers, Francia, siglo XI), las muestras analizadas para identificar los materiales indican la presencia del pigmento que se alternaba con lapislázuli aplicado en las zonas de mayor relevancia iconográfica.

Todos estos datos indican que el comercio de este tipo de pigmento estaba generalizado y que de hecho era considerado un pigmento de menor importancia que se destinaba a fondos, utilizándose pigmentos más caros como el lapislázuli para las figuras más importantes.

Sin embargo, la analítica material de San Martín de Elines (Cantabria) presenta una segunda extrañeza fuera de contexto: la presencia en el mortero de trazas de áridos de origen volcánico (basalto) en una zona de rocas calizas y sedimentarias donde se encuentra San Martín de Elines. Esto o relaciona materialmente las pinturas con autores de los Pirineos o indica que junto al conocido comercio de pigmentos existía también el de áridos que quizás aportaban determinadas características al soporte.

\section{- Herramientas que sirven para definir contexto}

El objetivo de este trabajo es establecer la necesidad de poner en práctica una metodología que facilite la relación entre bienes culturales, en este caso pintura mural románica. La normalización de esta acción facilitaría la posibilidad de generar productos encaminados al disfrute, relacionado con el conocimiento, y el deseo de preservación de los bienes que son conocidos y valorados en conjunto.

Con el objeto de dar continuidad al caso de intervención expuesto en San Martín de Elines, y dado que la problemática surge en el intento de relacionar estas pinturas con otras, el ejemplo de normalización que se expondrá se enmarcará en torno a la pintura mural románica, aunque como se ha comentado esta carencia es genérica en cualquier intervención en bienes culturales e impide posteriormente un procedimiento de cotejo.

Esta metodología está claramente definida por normativas internacionales. Sin embargo, como hemos comentado con anterioridad, no se encuentra totalmente implantada ni homogéneamente normalizada, lo que impide que surjan sinergias interdisciplinares y transdisciplinares. A pesar de este panorama, existen intentos incipientes de normalización como los promovidos por el Instituto de Patrimonio Histórico Andaluz (IPHA). ${ }^{3}$

En los últimos años aparentemente se comienza a dar solución al problema, pero se está lejos de adoptar unos criterios mínimos y comunes. Las pautas están definidas pero su implementación es lenta. Sin un organismo o institución que planifique una estrategia transversal que sirva para definir qué función deben tener los bienes culturales, el camino de la preservación desaparece. Su misión se diluye al no encontrar la forma de transferir el conocimiento para que la sociedad sienta que esta diferencia y variedad cultural es la que puede dar riqueza y singularidad a múltiples productos.

A nivel nacional, muchos son los esfuerzos que se están desarrollando para la consulta en red de fuentes documentales, catálogos y bases de datos de museos y bienes con declaración de protección. Todo ello marca una tendencia hacia lo que podría ser esta definición del contexto. Se pueden mencionar distintos ejemplos que van en esta línea como el Portal de Archivos Españoles (PARES), la Red Digital de Colecciones de Museos de España (CER.ES), el directorio de Museos y Colecciones de España. Determinadas comunidades se encuentran también implementando recientemente tecnología de búsqueda automática en línea. Por ejemplo el Visor Geográfico de Bienes Culturales de la Junta de Castilla y León [http://www.idecyl.jcyl.es/VisorLigeroPACU/ index2.html], o el buscador de nombres geográficos de Andalucía [http://www.ideandalucia.es/nomenclator/], o el incipiente proyecto del Instituto de Patrimonio Cultural Español (IPCE) "Archivo y Digitalización de muestras históricas de pintura y policromía de los laboratorios del IPCE" (ARCHIMUPAC).

Sin embargo, para el caso que nos ocupa no existe una herramienta relacional adecuada que nos indique siquiera determinados aspectos esenciales como la localización cartográfica de diferentes conjuntos. Por ejemplo, una herramienta que simplemente localizase la presencia de iglesias románicas. En relación con este ámbito el que más se aproxima es el proyecto de la Fundación Santa María la Real, de Aguilar de Campóo, Centro de Documentación de Arte Románico (CEDAR) [http://www.romanicodigital. com/Default.aspx]. No obstante la web de la fundación carece de la posibilidad de consulta geográfica, en la que "simplemente" poder lanzar la pregunta: [Iglesias románicas (S.XII)\Con o sin categoría de protección\ Localizadas en la zona norte de Burgos y Palencial Con restos de pintura mural $\backslash]$

Si, además, se pudiese profundizar con la consulta: [Con presencia de pigmento azul/ Que haya sido determinado mediante analítica], extrapolándolo a otras comunidades limítrofes como Cantabria, La Rioja, Aragón y Cataluña sería realmente sencillo establecer relaciones en el tema de ejemplo que nos ocupa. 


\section{Propuesta}

Analizadas las carencias, esta propuesta parte del análisis tipológico para mostrar un ejemplo de lo que podría ser la clasificación de pintura mural románica.

El objeto de esta identificación tipológica no es otro que el de facilitar la funcionalidad del bien, en cuanto a su capacidad de relacionarlo con otros similares, de forma que puedan ser apoyo en procesos de desarrollo económico y social en las zonas donde se ubican. Para ello será necesario realizar el análisis de composición material y técnica de ejecución, análisis de su distribución dentro de áreas territoriales determinadas, identificar procesos productivos característicos de determinados colectivos sociales, determinar cambios y permanencias en técnicas de ejecución, detectar las fórmulas de transmisión empleadas (aprendiz-maestros), establecer posibles parámetros de movilidad, establecer posibles relaciones sociales y cotejar los datos con fuentes documentales o bibliografía publicada.

El ámbito territorial debe basarse en criterios extensivos y abiertos desligados de los actuales límites territoriales y más vinculados con posibles vías de comunicación existentes en el período cronológico de estudio. No obstante, esta clasificación por zonas no debe ser decisiva ni condicionante, sino punto de partida para la selección de unidades territoriales que puedan relacionar obras que carecen de contexto y dataciones absolutas al no existir relaciones con fuentes primarias documentales.

Como posible ejemplo de recogida de datos para pintura mural románica se puede plantear el siguiente esquema [tabla 5 a y b]:

\begin{tabular}{|c|c|c|}
\hline \multicolumn{3}{|l|}{ IDENTIFICACIÓN } \\
\hline \multirow[t]{2}{*}{ LOCALIZACIÓN } & \multicolumn{2}{|c|}{ DATOS DE IDENTIFICACIÓN } \\
\hline & DE LAS MUESTRAS & DE LA EJECUCIÓN \\
\hline $\begin{array}{l}\text { - Provincia } \\
\text { - Municipio } \\
\text { - Inmueble } \\
\text { - Comarca histórica } \\
\text { - Edafología } \\
\text { - Corriente tipológica } \\
\text { - Posible época de } \\
\text { ejecución } \\
\text { - Imagen identifi- } \\
\text { cativa } \\
\text { - Bibliografía }\end{array}$ & $\begin{array}{l}\text { - Localización } \\
\text { - Original o repinte } \\
\text { - Color } \\
\text { - Textura (lisa o } \\
\text { rugosa) } \\
\text { - Secuencia de capas } \\
\text { - Representación } \\
\text { - Iconográfica (dado } \\
\text { que determinados } \\
\text { pigmentos aparecen } \\
\text { localizados en deter- } \\
\text { minadas imágenes) }\end{array}$ & $\begin{array}{l}\text { - Técnica de ejecu- } \\
\text { ción (fresco, seco, } \\
\text { técnica mixta, otras } \\
\text { hipótesis) } \\
\text { - Existencia de } \\
\text { sinopia } \\
\text { - Marcas de ejecución } \\
\text { - Características } \\
\text { físicas del mortero } \\
\text { (color, granulometría) }\end{array}$ \\
\hline
\end{tabular}

Tabla 5. a.- Propuesta de ficha para recogida de datos. Fuente: autor. @Rosa Tera

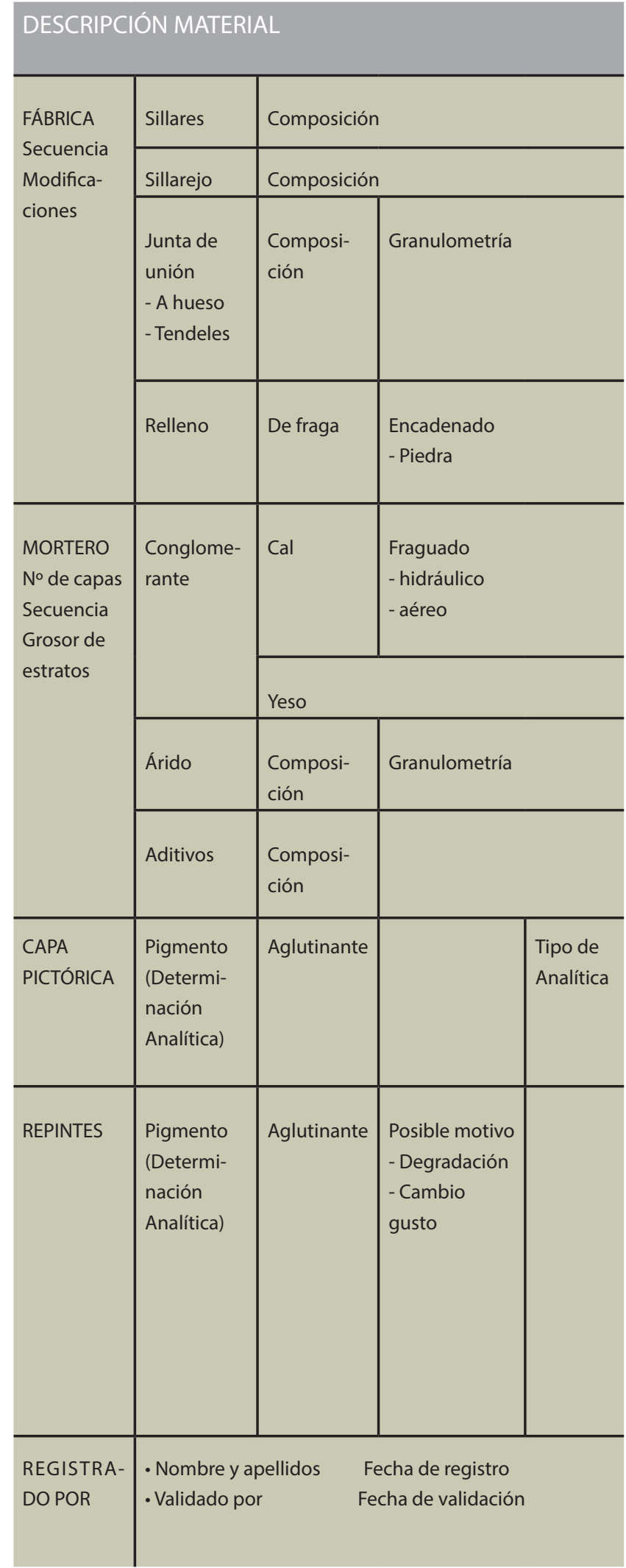

Tabla 5. b.- Propuesta de ficha para recogida de datos. Fuente: autor. @Rosa Tera

La recogida de datos de la anterior ficha, por ejemplo, podrá dar como resultado las siguientes tipologías técnicas [tabla 6]: 


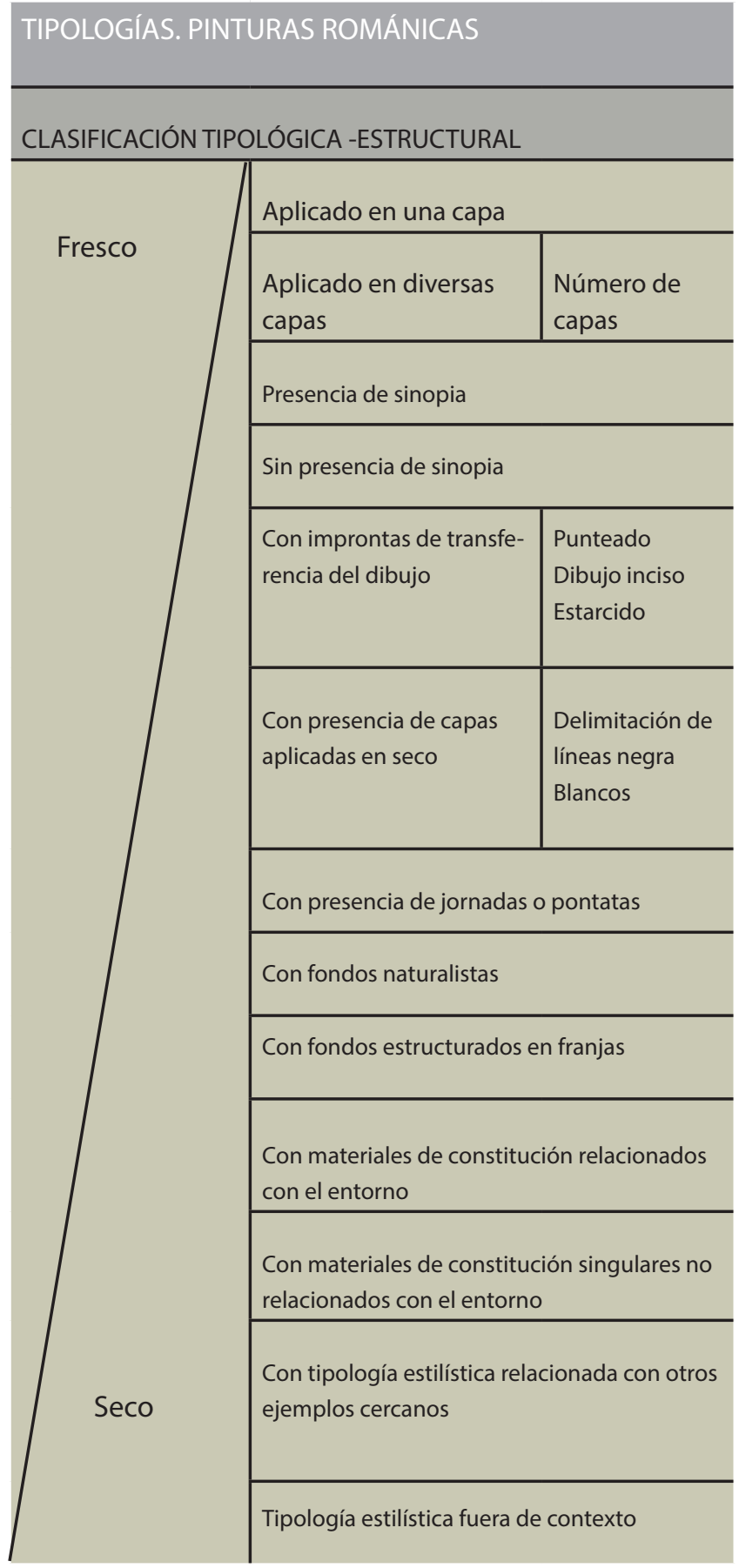

Tabla 6.- Propuesta de ficha para recogida de datos. Fuente: autor. @Rosa Tera

Esta clasificación de tipologías estructurales podría luego relacionarse en un Sistema de Información Geográfico mediante capas que reflejasen delimitaciones administrativas históricas, antiguas vías de comunicación o zonas que han tenido en determinado momento relaciones con determinados enclaves o formas de gobierno. Esto posibilitaría, quizás que, a falta de fuentes primarias de información, se pudiesen establecer relaciones que indicasen los contextos sociales, relaciones o movilidad, por los que se pudieron desarrollarse los distintos conjuntos pictóricos.
Este sistema de ordenación de la información, abierta en red y normalizada, permitiría almacenar, editar, analizar, compartir y mostrar la información geográficamente relacionada, al mismo tiempo que realizar consultas interactivas, editar datos y presentar los resultados en mapas en beneficio de proyectos de investigación y desarrollo territorial relacionados con cultura y turismo.

\section{Conclusiones}

Los materiales constitutivos de las pinturas murales de San Martín de Elines (Cantabria) muestran dos evidencias fuera del contexto geológico de areniscas donde se ubican.

La identificación de roca basáltica en el mortero de las pinturas, y la presencia del mineral aerinita como pigmento está relacionados con mapas geológicos que se circunscribe en la península Ibérica a la zona del Pirineo. La aerinita es un pigmento escaso que pudo haberse extendido por medio del comercio, sin embargo el hecho de que en el mortero de las pinturas además aparezca basalto como árido nos está remitiendo a procesos migratorios de artistas o talleres.

Para poder demostrar estas relaciones es necesario normalizar el sistema de análisis de materiales y garantizar que esta información sea accesible y de libre consulta, con el fin de poder facilitar el estudio comparativo entre manifestaciones constructivas de distintas zonas geográficas.

La importancia de numerosos datos técnicos que se relacionan con intervenciones de patrimonio cultural transciende el sentido de aquellos que son necesarios para la intervención. Su funcionalidad radica en la posibilidad de establecer contextos relacionales que puedan indicar particularidades y semejanzas que poseen unas obras respecto a otras. Si no se pueden establecer este tipo de enlaces, los datos que nos ayuden a definir su contexto específico aparecerán como anecdóticos.

\section{Notas}

[1] El clero francés, reforzado por el origen borgoñés del primer marido de Urraca, temía perder los privilegios. Con el matrimonio se anulaban, también, los derechos al trono castellano-leonés del hijo de Urraca, Alfonso Raimúndez, y los nobles castellano-leoneses veían amenazado su poder con el nombramiento de cargos importantes a favor de nobles aragoneses.

[2] En 1116 Alfonso I concede fuero a Belorado y hasta las Paces de Támara 1127 su vasallo Sancho de Arnáldez domina la ciudad de Burgos e incluso después la plaza de Castrogeriz.

[3]En el año 2004 en la revista del Instituto de Patrimonio Histórico Andaluz (IPHA), publicada por el Centro de 
Intervención en el Patrimonio Histórico de Andalucía aparece el artículo "Metodología para la intervención en el patrimonio histórico: normalización de la documentación", donde se indica la apertura de otros protocolos como: "Protocolo de normalización de intervenciones en el Patrimonio Histórico", donde quedan abiertos otros protocolos como el de "Publicación de las memorias de memorias de las intervenciones", "Normalización de Informes de Paleobiología", "Normalización de Informes Analíticos", y“Normalización de Informes de Conservación Preventiva".

\section{Bibliografía}

BELLINI, C. y GARRETA, M. (2001). "Las Teorías en Arqueología", La Trama Cultural. Textos de Antropología. Buenos Aires: Caligraf, 137-145.

CARL, C. (2008). "Munio, obispo de Calahorra, 1066 a 1080 ¿Defensor del rito mozárabe? Una revisión de las pruebas documentales", Revista Hispania Sacra, LX, 122: 685-701.

DANIEL, F. et all (2008). "Le pigment d'aérinite dans deux peintures murales romanes du Sud-Ouest de la France ", ArchéoSciences. Revue d'Archéométrie [Online], 32, http://archeosciences.revues.org/987 [consulta: 23/01/2012]

FERRER MORALES, A. (1998): La pintura mural. Su soporte, conservación, restauración y las técnicas modernas, Universidad de Sevilla, Sevilla.

FUNDACIÓN SANTA MARÍA LA REAL, Románico Digital, http://www.romanicodigital.com/Default.aspx[consulta: 23/01/2012]

GARCÍA GUINEA, M.A. y PÉREZ GONZALEZ, J.M. (2007). Enciclopedia del románico en Cantabria T.3. Campoo, Los Valles. Aguilar de Campoo. Aguilar de Campóo (Palencia): Fundación Santa María la Real, Centro de Estudios del Románico.

GASOL, R. M. (2007): Propuesta metodológica de estudio de la pintura mural en Cataluña. Aplicación a diversos conjuntos fechados entre los siglos X-XIX , III Congreso del Grupo Español del IIC.

HAWTHORNE, J. G. y SMITH, C. S. (1979). "The first book: The art of the painter", OnDivers Arts: the foremost mediaeval treatise on painting, glassmaking and metalwork. New York: Dover ,11-43.

HUERTA HUERTA, P.L. (2004). "Revestimientos polícromos y pinturas murales en el románico Burgalés". En El arte románico en el territorio Burgalés. RODRÍGUEZ PAJARES, E.J. (dir.). Burgos: Universidad Popular para la Educación y Cultura de Burgos, 206
JUNTA DE ANDALUCÍA. INSTITUTO DE ESTADÍSTICA Y CARTOGRAFÍA DE ANDALUCÍA. CONSEJERÍA DE ECONOMÍA, INNOVACIÓN Y CIENCIA, Buscador de nombres geográficos, http://www.ideandalucia.es/ nomenclator/[consulta: 23/01/2012]

JUNTA DE CASTILLA Y LEÓN, Bienes Culturales de Castilla y León (BICUcy), http://www.idecyl. jcyl.es/VisorLigeroPACU/index2.html[consulta: 23/01/2012]

PALET CASAS, A. y DE ANDRES LLOPIS, J. (1992). "The identification of aerinite as a blue pigment in the romanesque frescoes of the pyrenean region", Studies in Conservation, Vol. 37, $n^{\circ} 2$. International Institute for Conservation of Historic and Artistic Works (IIC), 132136.

PARRA GRECO, E. (2011). Informe analítico pinturas murales de San Martín de Elines (Cantabria) para la empresa Batea Restauraciones.

PORTA, E.; PALET, A. y GUILAMET, E (1993). "Le bleu aérinite: un pigment méconnu en peinture murale romane". En $X$ réunion triennale de I'ICOM-CC, vol. 2. ICOM-CC, 534-538.

RIUS, J. (2004): “El misterioso azul del románico catalán", R+D CSIC, 02-02-2004, en línea www.ott.csic.es/rdcsic/ rdciscesp/rdma20esp.htm [Consulta 16-04-13]

SÁINZ RIPA, E. (1990). "Los obispos de Calahorra en el edad media (siglos VIII-XV)". En I Semana de Estudios Medievales. IGLESIA DUARTE, J.I. (coord.). Nájera, Instituto de Estudios Riojanos, 37-66.

V.V.A.A. (2000). “Carta internacional sobre los principios para la conservación y la restauración de patrimonio construido". En Conferencia Internacional sobre Conservación. Cracovia. http://ipce.mcu.es/pdfs/2000_ Carta_Cracovia.pdf [consulta: 23/01/2012]

V.V.A.A. (2004). "Metodología para la intervención en el patrimonio histórico: normalización de la documentación". Revista PH: Boletín del Instituto Andaluz del Patrimonio Histórico, Año n¹2, n47. Junta de Andalucía: Instituto Andaluz del Patrimonio Histórico, 71-83.

WETTSTEIN, J. (1971). La fresque romane. Italia-FranceEspagne. Études comparatives.Vol.1. Italie, France, Espagne. Genève: Droz. 


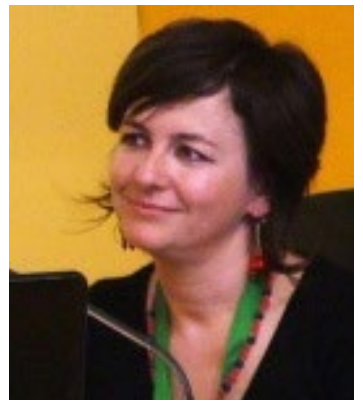

\section{Rosa Tera Saavedra}

rosatesa@gmail.com

Licenciada en Bellas Artes por la Universidad de Granada en la especialidad de Conservación y Restauración pictórica.. Master en Patrimonio Histórico y Territorial por la Universidad de Cantabria. Empresaria socia de Batea S.L., desde 1997. En la actualidad desarrolla proyectos de gestión patrimonial que pretenden el desarrollo social basado en valores patrimoniales

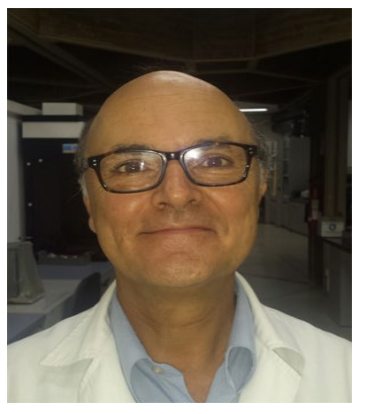

\section{Enrique Parra Crego}

larcoquimica@hotmail.com

Doctor en Química por la Universidad Autónoma de Madrid en 1992. Regenta la empresa de análisis Larco Química y Arte S.L. desde 2004. Ha trabajado en el Instituto de Conservación y Restauración de Bienes Culturales de 1988 a 1994, profesor de análisis químico en la Universidad Alfonso X el Sabio de 1994 a 2012 y vicerrector de investigación de 1997 a 2007 . Actualmente en el Instituto del Patrimonio Cultural de España en el Área de Investigación 